\section{(6) OPEN ACCESS}

\title{
Epidemiology of genital warts in the British population: implications for HPV vaccination programmes
}

\author{
Pam Sonnenberg, ${ }^{1}$ Clare Tanton, ${ }^{1,2}$ David Mesher, ${ }^{3}$ Eleanor King, ${ }^{1}$ Simon Beddows, ${ }^{4}$ \\ Nigel Field, ${ }^{1}$ Catherine H Mercer, ${ }^{1}$ Kate Soldan, ${ }^{3}$ Anne M Johnson ${ }^{1}$
}

${ }^{1}$ Mortimer Market Centre, Institute for Global Health, University College London, London, UK

${ }^{2}$ Department of Infectious Disease Epidemiology, London School of Hygiene \& Tropical Medicine (LSHTM), London, UK ${ }^{3}$ Centre for Infectious Disease Surveillance and Control (CIDSC), Public Health England, London, UK

${ }^{4}$ Virus Reference Department, Public Health England, London, UK

\section{Correspondence to}

Professor Pam Sonnenberg, Mortimer Market Centre Institute for Global Health, University College London, London WC1E 6JB, UK; p. sonnenberg@ucl.ac.uk

Received 30 July 2018 Revised 6 November 2018 Accepted 25 November 2018 Published Online First 5 February 2019

\section{Linked}

- http://dx.doi.org/10.1136/ sextrans-2018-053751

Check for updates

(C) Author(s) (or their employer(s)) 2019. Re-use permitted under CC BY. Published by BMJ.

To cite: Sonnenberg $P$ Tanton C, Mesher D, et al. Sex Transm Infect 2019:95:386-390.

\begin{abstract}
Objectives To estimate the prevalence of, and describe risk factors for, genital warts (GWs) in the British population, following the introduction of the bivalent (human papillomavirus (HPV)-16/18) vaccination programme in girls, and prior to the switch to quadrivalent (HPV-6/11/16/18) vaccine (offering direct protection against GWs) and compare this with GW diagnoses in the prevaccination era.

Methods Natsal-3, a probability sample survey in Britain, conducted in 2010-2012, interviewed 9902 men and women aged 16-44. Natsal-2, conducted in 1999-2001, surveyed 11161 men and women aged 16-44. Both surveys collected data on sexual behaviour and sexually transmitted infection diagnoses using computer-assisted interview methods.

Results In Natsal-3, 3.8\% and $4.6 \%$ of sexually experienced men and women reported ever having a diagnosis of GWs, with $1.3 \%$ of men and $1.7 \%$ of woman reporting a GWs diagnosis in the past 5 years. GWs were strongly associated with increasing partner numbers and condomless sex. Diagnoses were more frequent in men who have sex with men (MSM) $(11.6 \%$ ever, $3.3 \%$ past 5 years) and in women reporting sex with women (10.8\% ever, $3.6 \%$ past 5 years). In the age group who were eligible for vaccination at the time of Natsal-3 (16-20 years), a similar proportion of sameaged women reported a history of GWs in Natsal-2 $(1.9 \%, 1.1-3.4)$ and Natsal-3 $(2.6 \%, 1.5-4.4)$.

Conclusions These data provide essential parameters for mathematical models that inform cost-effectiveness analyses of HPV vaccination programmes. There was no evidence of population protection against GWs conferred by the bivalent vaccine. Even with vaccination of adolescent boys, vaccination should be offered to MSM attending sexual health clinics.
\end{abstract}

\section{INTRODUCTION}

Genital warts (GWs) are the most common viral sexually transmitted infection (STI) diagnosed among genitourinary medicine clinic (GUM) attendees in the $\mathrm{UK}^{1}$ and globally. ${ }^{2}$ The diagnosis and treatment of GWs also has associated individual and healthcare costs: in 2008, the annual expenditure on GWs in England was estimated at $£ 16.8$ million, ${ }^{3}$ and each episode is estimated to have a QALY loss equivalent to 6.6 days of healthy life lost. ${ }^{4}$
In 2008, the UK began a human papillomavirus (HPV) immunisation programme in adolescent girls (aged 11-12 years, with catch-up to 17 years), initially using the bivalent vaccine which protects against HPV types 16 and 18, the most frequent causes of cervical cancer. In 2012, the programme switched to quadrivalent HPV vaccine which additionally protects against HPV types 6 and 11, which are responsible for over $90 \%$ of cases of GWs. ${ }^{5}$

A recent systematic review ${ }^{6}$ of the effectiveness of the quadrivalent vaccine, following 10 years of real-world experience, reported that, as early as 2 years after starting a vaccination programme in girls, and with high coverage such as in Australia and Denmark, there was a rapid and marked reduction in the incidence of GWs among women eligible for vaccination and some benefit conferred to similar-aged heterosexual men. ${ }^{7}$ Reductions in GWs in men who have sex with men (MSM) have been, as expected, less evident. ${ }^{8}$ In England, surveillance data from GUM clinics have shown an unexpected reduction in diagnoses of GWs between 2009 and $2011,{ }^{9}$ particularly in women in the age groups offered bivalent vaccination and, to a lesser extent, in similar-aged heterosexual men, and this has continued to $2014 .{ }^{10}$ This ecological observation, together with findings of moderate efficacy against some low-risk HPV types in a posthoc analysis of the PATRICIA (Papilloma TRIal against Cancer In young Adults) trial of the bivalent vaccine, ${ }^{11}$ has led to a hypothesis that the bivalent vaccine may confer a modest cross-protective effect against GWs.

The availability of population-based comparison data from the prevaccination era is important to demonstrate population impact. ${ }^{6}$ Furthermore, it is important to monitor the prevalence of GWs in different subgroups, to ensure that vaccination programmes are widely accessed, reach those at greatest risk and reduce health inequalities. For Britain, the National Surveys of Sexual Attitudes and Lifestyles (Natsal) are able to capture the population burden of STIs and link this to detailed behavioural information. This includes people who access services for testing, diagnosis and treatment including, but not limited to sexual health (GUM) clinics, which are the main contributors to STI surveillance. The timings of the Natsal surveys allow estimation of the population effects of HPV vaccination: Natsal-2 was conducted in 1999-2001 
prior to the introduction of any HPV vaccination, with Natsal-3 in 2010-2012 taking place after the introduction of bivalent vaccination (but prior to the switch to quadrivalent vaccine).

This paper reports the prevalence of reported diagnoses of, and risk factors for, GWs in the British population in Natsal-3. We examine changes in reported prevalence of GW diagnoses between the birth cohorts eligible for vaccination in Natsal-3, with those of equivalent age in the prevaccination era in Natsal-2, to assess whether there is population-based evidence of cross-protection against GWs.

\section{MATERIALS AND METHODS Participants and procedures}

Natsal-3 interviewed 15162 men and women aged 16-74 in 2010-2012. Details of the study methods have been described previously. ${ }^{12}$ Briefly, we used a multistage, clustered, stratified probability sample design. The response rate was $57.7 \%$. Participants were interviewed through a combination of face-to-face computer-assisted personal interview and computer-assisted self-interview for the more sensitive questions, including on their experience of STI diagnoses. Participants were asked if they had ever been told by a doctor or other healthcare professional that they had any of a list of different STIs, including GWs, and if so, when they were last diagnosed with GWs. Natsal-2 (undertaken in 1999-2001 surveyed 11161 men and women aged 16-44, with a response rate of $65.4 \% .{ }^{13}$ The surveys use similar methodology to allow comparison over time. HPV catch-up vaccination coverage in Natsal-3 participants was $61.5 \% .{ }^{14}$ Vaccine uptake varied by school year at eligibility, with $72.9 \%$ of women eligible at 14 years reporting having received all three doses, compared with only $50.6 \%$ of women eligible at 17 years. $^{15}$

\section{Statistical analysis}

Of the 3809 men and 5510 women aged 16-44 years in Natsal-3, we included sexually experienced participants with information on GW history (3570 men (93.7\%) and 5257 women (95.4\%)) in the main analysis. Analysis accounts for the stratification, clustering and weighting of the sample. We describe the prevalence of reported history of GWs over different time periods by age. In those reporting at least one partner in the past 5 years (of either gender), we stratify this according to experience of same-sex sex during this timeframe. Age-adjusted odds ratios (aORs) are calculated for the association between key behavioural risk factors and a history of GWs in the past 5 years. Data for comparison between Natsal-2 (4267 men and 5869 women) and Natsal-3 were available for sexually experienced men and women aged 16-44 years. We describe the prevalence of reported history of GWs in the age group of the birth cohorts in Natsal-3 who would have been eligible for vaccination (aged 16-20 years) and present a prevalence ratio with $95 \%$ CIs. Data were analysed using Stata (V.14.1).

\section{Ethics}

Natsal-3 was granted ethical approval by the Oxfordshire Research Ethics Committee A (Reference: 09/H0604/27). Natsal-2 obtained ethical approval from University College Hospital, North Thames Multicentre and all local research ethics committees in Britain.

\section{RESULTS}

Using data from Natsal-3 (2010-2012), table 1 shows how the proportion of the population reporting a diagnosis of GWs varied by age and gender. Overall, $3.8 \%$ of sexually experienced men and $4.6 \%$ of sexually experienced women aged $16-44$ had ever had a diagnosis of GWs. Ever diagnosis increased with age, plateauing at around 5\% at age 25 in men and age 20 in women. In total, 1.3\% of men and $1.7 \%$ of women had a diagnosis in the past 5 years, with $0.3 \%$ of men and women reporting a diagnosis in the past year.

Diagnoses were more frequent in MSM $(11.6 \%$ ever and $3.3 \%$ past 5 years) than men who reported exclusively having sex with women (3.6\% ever and $1.2 \%$ past 5 years) (table 2 ). Similarly, diagnoses were more frequent in women reporting sex with women (WSW) (10.8\% ever and $3.6 \%$ past 5 years) than in women who reported exclusively having sex with men $(4.3 \%$ and $1.6 \%$, respectively).

A diagnosis of GWs was associated with age, but there were no significant associations with ethnicity, area-level deprivation or educational status (data not shown). Figure 1 shows behavioural factors associated with a diagnosis of GWs in the past 5 years in those with one or more partner in this timeframe. For both men and women, a warts diagnosis was strongly associated with increasing partner numbers and condomless sex.

Those reporting a same-sex partner in the past 5 years were also more likely to report a history of GWs (aOR $2.66(0.94$ $7.50)$ in men and 1.99 (1.00-3.94) in women); however, this effect was no longer significant after adjusting further for partner

Table 1 Reported diagnoses of genital warts, ever, in the past 5 years and in the past year, in British men and women aged 16-44*

\begin{tabular}{|c|c|c|c|c|c|c|c|c|c|c|}
\hline & \multicolumn{2}{|l|}{$16-19$} & \multicolumn{2}{|l|}{$20-24$} & \multicolumn{2}{|l|}{$25-34$} & \multicolumn{2}{|l|}{$35-44$} & \multicolumn{2}{|c|}{ Total aged $16-44$} \\
\hline & $\%$ & $95 \% \mathrm{Cl}$ & $\%$ & $95 \% \mathrm{Cl}$ & $\%$ & $95 \% \mathrm{Cl}$ & $\%$ & $95 \% \mathrm{Cl}$ & $\%$ & $95 \% \mathrm{Cl}$ \\
\hline \multicolumn{11}{|l|}{ Men } \\
\hline Denom. (unwt, wt) ${ }^{\prime}$ & 578,370 & & 785,620 & & 1432,1284 & & 775,1367 & & 3570,3642 & \\
\hline \multicolumn{11}{|l|}{ Genital warts } \\
\hline Ever & $0.2 \%$ & $(<0.1$ to 0.9$)$ & $1.8 \%$ & (1.0 to 3.3 ) & $4.7 \%$ & (3.6 to 6.1 ) & $4.9 \%$ & (3.4 to 6.8 ) & $3.8 \%$ & (3.0 to 4.7 ) \\
\hline Past 5 years & $0.2 \%$ & $(<0.1$ to 0.9$)$ & $1.8 \%$ & (1.0 to 3.3 ) & $2.0 \%$ & (1.3 to 3.0$)$ & $0.6 \%$ & (0.3 to 1.5 ) & $1.3 \%$ & (0.9 to 1.7$)$ \\
\hline Past year & $0.2 \%$ & $(<0.1$ to 0.9$)$ & $0.4 \%$ & (0.1 to 1.4 ) & $0.4 \%$ & (0.1 to 1.1$)$ & $0.2 \%$ & $(<0.1$ to 1.3$)$ & $0.3 \%$ & (0.2 to 0.6$)$ \\
\hline \multicolumn{11}{|l|}{ Women } \\
\hline Denom. (unwt, wt) & 667,341 & & 1059,621 & & 2362,1304 & & 1169,139 & & 5257,3662 & \\
\hline \multicolumn{11}{|l|}{ Genital warts } \\
\hline Ever & $1.5 \%$ & (0.7 to 2.9 ) & $5.0 \%$ & (3.6 to 7.0 ) & $4.8 \%$ & (3.9 to 6.0 ) & $4.9 \%$ & (3.8 to 6.4 ) & $4.6 \%$ & (4.0 to 5.3 ) \\
\hline Past 5 years & $1.1 \%$ & (0.6 to 2.3 ) & $4.3 \%$ & (2.9 to 6.3 ) & $1.6 \%$ & (1.1 to 2.4 ) & $0.6 \%$ & (0.3 to 1.3 ) & $1.7 \%$ & (1.3 to 2.1$)$ \\
\hline Past year & $0.7 \%$ & (0.3 to 1.7 ) & $1.2 \%$ & (0.6 to 2.5 ) & $0.1 \%$ & (<0.1 to 0.6$)$ & $<0.1 \%$ & $(<0.1$ to 0.4$)$ & $0.3 \%$ & ( 0.2 to 0.6$)$ \\
\hline
\end{tabular}

${ }^{*}$ Data from Natsal-3 2010-2012.

tDenominators are those with $1+$ partner ever. 
Table 2 Reported diagnoses of genital warts in those aged 16-44 years by same-sex behaviour in the past 5 years*

\begin{tabular}{|c|c|c|c|c|c|c|c|c|}
\hline & \multicolumn{4}{|l|}{ Men } & \multicolumn{4}{|l|}{ Women } \\
\hline & \multicolumn{2}{|l|}{ MSEW† } & \multicolumn{2}{|l|}{ MSMT } & \multicolumn{2}{|l|}{ WSEMT } & \multicolumn{2}{|l|}{ wsWt } \\
\hline & $\%$ & $95 \% \mathrm{Cl}$ & $\%$ & $95 \% \mathrm{Cl}$ & $\%$ & $95 \% \mathrm{Cl}$ & $\%$ & $95 \% \mathrm{Cl}$ \\
\hline Denom. (unwt, wt)‡ & 3377,3471 & & 137,116 & & 4881,3430 & & 304,189 & \\
\hline \multicolumn{9}{|l|}{ Genital warts } \\
\hline Ever & $3.6 \%$ & $(2.9-4.4)$ & $11.6 \%$ & $(5.7-22.1)$ & $4.3 \%$ & $(3.6-5.0)$ & $10.8 \%$ & $(7.2-15.8)$ \\
\hline Past 5 years & $1.2 \%$ & $(0.9-1.7)$ & $3.3 \%$ & $(1.2-8.3)$ & $1.6 \%$ & $(1.2-2.0)$ & $3.6 \%$ & $(2.0-6.5)$ \\
\hline Past 1 year & $0.3 \%$ & $(0.1-0.6)$ & $0.4 \%$ & $(0.0-2.5)$ & $0.3 \%$ & $(0.2-0.6)$ & $0.7 \%$ & $(0.2-2.6)$ \\
\hline
\end{tabular}

${ }^{*}$ Data from Natsal-3 2010-2012.

tMSEW (men who have sex exclusively with women); MSM (men who have sex with men, including men who have sex with men and women).

$\neq$ Denominators are those with $1+$ partner in the past 5 years.

WSEM, women who have sex exclusively with men; WSW, women who have sex with women, including women who have sex with men and women.

numbers (adjusted OR $0.95(0.29-3.13)$ in men and $0.92(0.46-$ 1.84) in women).

In Natsal-2, data were only available on a history of GWs ever. This was reported in 3.7\% (3.1\%-4.3\%) of men and $4.2 \%$ $(3.7 \%-4.8 \%)$ of women aged $16-44$ years, very similar proportions to those reported a decade later in Natsal-3. Specifically, in women in the birth cohorts who would have been eligible for vaccination in Natsal-3 (16-20 years at time of interview), a similar proportion of same-aged women reported a history of GWs in Natsal-2 (13/556, weighted prevalence 1.9\%, 1.1-3.4) and Natsal-3 (21/870, weighted prevalence 2.6\%, 1.5-4.4), with a prevalence ratio, comparing Natsal-3 to Natsal-2, of 1.35 (95\% CI: 0.61 to 2.98 ).

\section{DISCUSSION}

Repeated cross-sectional population-based decennial surveys, such as Natsal, can provide baseline and postintervention estimates of prevalence, behaviour and uptake of services for national programmes. These are vital to monitor impact, model cost-effectiveness and guide decision-making on interventions, including vaccination strategies. Natsal data complements surveillance but is also able to provide important parameters for modelling HPV transmission dynamics that cannot be ascertained from clinic-based data, such as lifetime GW diagnoses and detailed sexual behaviour.

A diagnosis of GW was associated with markers of more risky sexual behaviours, similar to those for other STIs, including high-risk HPV infection. ${ }^{14}$ GWs were less common in heterosexual men and women in the general population, compared with men and women reporting same-sex behaviour. During the same time period as Natsal-3, a cross-sectional survey of $\sim 500$ MSM attending a London GUM clinic was undertaken. ${ }^{16}$ In this clinic-attending population of MSM, nearly one-third had a previous diagnosis of GWs ever (30.3\%), 1 in 10 had a GWs

Same-sex partner, past 5y

Number of partners, past $5 y$

Number of partners without a condom, past $5 y$

Men

(unwt=3553, wt $=3631$ )

Age

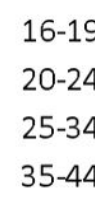

$16-19$
$20-24$
$25-34$
$35-44$

No

Yes

1

2

3-4

$5+$
0
1
$2+$

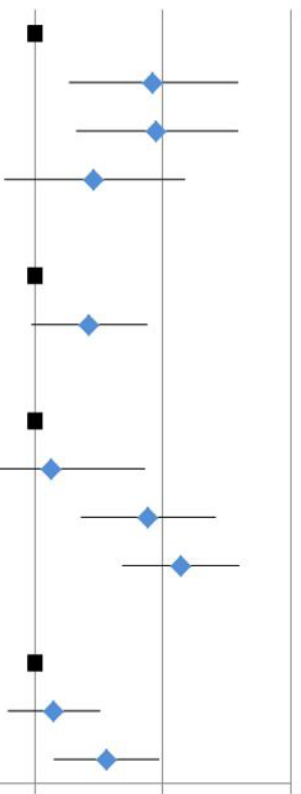

1
Women

(unwt $=5218, w t=3640$ )

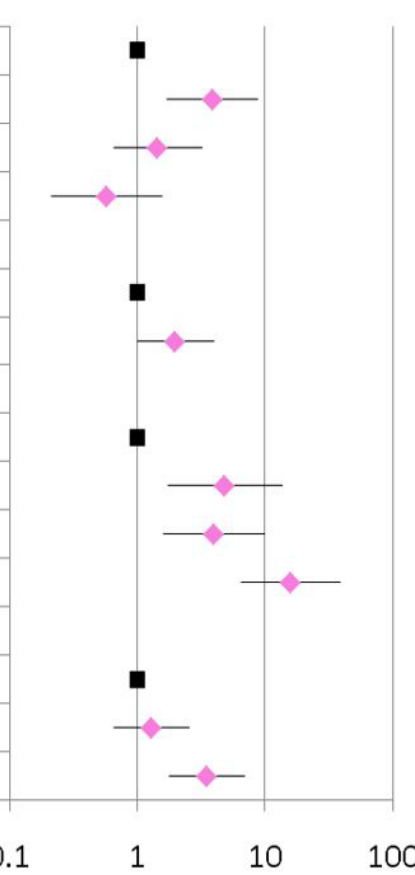

Figure 1 Factors associated with a diagnosis of genital warts in the past 5 years, in men and women aged 16-44. * See separate jpg file. *Data from Natsal-3. denominators are those with 1+partner in the past 5 years. Age-adjusted ORs and $95 \% \mathrm{Cls}$. 
diagnosis in the past year $(9.8 \%)$ and in many cases they had had more than one episode. ${ }^{17}$ Our finding is consistent with knowledge that MSM have a higher incidence of HPV infection and related disease. ${ }^{18}$ This knowledge, along with the expectation that MSM will benefit less from herd protection from the vaccination of women, informed the Joint Committee on Vaccination and Immunisation's (JCVI) advice in 2016 that quadrivalent vaccination should be offered to MSM attending sexual health and HIV clinics, as a cost-effective intervention. ${ }^{19}$ Following this advice, a pilot of HPV vaccination for MSM attending sexual health and HIV clinics across England was introduced in summer 2016, with similar vaccination programmes introduced nationally in Wales (since April 2017) ${ }^{20}$ and Scotland (since July 2017). ${ }^{21}$ The pilot showed feasibility and acceptability $(45.5 \%$ recorded first dose uptake) and a national HPV vaccination programme for MSM (up to and including 45 years of age) is being rolled-out from April 2018. ${ }^{22}$ The recommendation to offer vaccination up to this age is based on the knowledge that while the vaccine may be more effective in those without previous HPV exposure (who are likely to be younger), the majority of MSM attending clinics have not been infected with all HPV-types in the vaccine ${ }^{16}$ and high-risk sexual behaviour, which is not restricted to young MSM, may result in repeated exposure.

Of note is that the prevalence estimates of ever having a diagnosis of GWs in MSM and WSW in the general population were similar at $\sim 11 \%$. The numbers of women who have sex exclusively with women in Natsal are very small, and the higher prevalence in WSW is likely to be in women having sex with both men and women. WSW have increased risk of STIs, higher partner numbers and other high-risk behaviours ${ }^{23}$ but the higher odds of GWs in WSW was no longer significant after adjusting for partner numbers. Nevertheless, given the high prevalence of GWs as well as lower uptake of cervical screening, ${ }^{15}$ it is important that WSW are included in health promotion messages and that vaccination coverage is high in this group. Women reporting more than five partners were at even higher risk (figure 1). This supports mop-up vaccination in these women if they attend sexual health services while in the eligible age group, if they had missed one or more doses in the routine programme.

Using data from Natsal-2 and Natsal-3, we have previously shown an early indication of population-based effectiveness of the bivalent HPV vaccine in women in the age groups eligible for vaccination, with a $\sim 50 \%$ reduction seen in the prevalence of HPV-16/18 in 18-20 year olds, but no observed change in prevalences of cross-protective types or in women aged $21-44 .{ }^{1424} \mathrm{We}$ have also previously reported that the prevalence of HPV-6/11 in urine in women in the age group eligible for vaccination had not reduced significantly (9.5\% in Natsal-2 vs $8.9 \%$ in Natsal-3, an age-adjusted prevalence ratio of $0.91(0.44-1.89)) .{ }^{2425}$ Likewise, in this paper, we found no change in the reporting of GW diagnoses following introduction of bivalent vaccination.

Our findings concur with the lack of protection against GWs observed in bivalent vaccine-immunised women in a number of studies ${ }^{26-28}$ and with the finding of no reduction in HPV-6 or HPV-11 infection in England ${ }^{29}$ or Scotland. ${ }^{30}$ Together, these data provide some evidence to refute the hypothesis of the HPV-16/18 vaccine conferring a cross-protective effect against HPV-6/11 and GWs. It is, however, possible that the timing of Natsal-3 relative to the start of the vaccination programme means that it was too soon to detect an effect on GWs and low risk HPV types, particularly since catch-up uptake was $\sim 60 \%$ (but early enough to ascertain the greater direct effect on HPV-16/18). ${ }^{14}$ Small numbers may also have resulted in insufficient power to detect a significant difference in the prevalence ratio. Reductions in GWs were seen in the surveillance data by 2011: possible reasons for this should have generated the same effects in Natsal-3, with the exception of changes in service use or other artefacts and measurement errors (when looking at diagnosis trends) in the surveillance data.

This paper presents data from Natsal-3, prior to the switch to quadrivalent vaccine. Based on data from other countries and that in the UK, there has already been high coverage of quadrivalent vaccine in girls for 5 years, large reductions in population HPV-6/11 prevalence, and in diagnoses of GWs in both women and men, are already expected. Indeed, this is the case: in 2017, the rate of first episode GWs diagnoses among females aged 15-17 attending specialist sexual health services was $89 \%$ lower compared with 2009 , with a decline of $70 \%$ in same aged heterosexual males over this time period, suggesting substantial herd protection. ${ }^{31}$ The impact of selective vaccination of MSM attending GUM/HIV clinics is expected to become evident in due course, as the vaccination programme started more recently and uptake is opportunistic and so will likely accrue more gradually. Following the recent JCVI recommendation to extend the adolescent girls programme to boys, ${ }^{32}$ even further reductions, in a shorter timeframe, are to be expected. As discussed above, even with a vaccination programme that includes adolescent boys, there remains a case for continuing to offer vaccination to MSM attending sexual health clinics up to age $45 .{ }^{19}$ In addition to the high burden of infection and disease, a large proportion of MSM attending clinics may not have been born in the UK (>50\% in the London study), ${ }^{16}$ including those from countries without a vaccination programme.

Cost-effectiveness analyses will continue to be needed to inform the UK HPV immunisation programme, for example, as new vaccines enter the market. Updating and extending this evidence is therefore helpful to reduce error and uncertainty within such analyses. These data may also be informative for other service and treatment planning, for example in countries considering introduction of, or changes to, a HPV vaccination programme. Further real-world evaluation of the impact of HPV vaccination on GWs should include monitoring in sexual health clinics, as part of routine surveillance and in stand-alone research studies, complemented by population-based surveys.

Key messages

- In the British population, 3.8\% of sexually experienced men and $4.6 \%$ of sexually experienced women aged $16-44$ reported ever having a diagnosis of genital warts (GWs).

- GWs were more common in men and women reporting same-sex behaviour ( $11 \%$ ever diagnosed with GWs).

- There was no evidence of the bivalent vaccine conferring a cross-protective effect against GWs.

- Cost-effectiveness analyses, and real-world evaluation, will continue to be needed to inform UK human papillomavirus (HPV) immunisation policy, as changes are made to the vaccination programme (eg, vaccinating boys) and new vaccines enter the market (eg, nonavalent HPV vaccine).

\section{Handling editor Jackie A Cassell}

Acknowledgements We thank the study participants, the team of interviewers from NatCen Social Research and operations and computing staff from NatCen Social Research.

Contributors PS, CT, DM, SB, NF and KS originally conceived this article. PS wrote the first draft, with extensive input from $C T$ and DM and further contributions from EK, SB, NF, CHM, KS and AMJ. CT and EK conducted the statistical analysis. AMJ, 
CHM and PS, initial applicants on Natsal-3, wrote the study protocol and obtained funding. PS, CT, SB, NF, CHM, KS and AMJ designed the Natsal-3 questionnaire, applied for ethics approval, undertook piloting and managed the fieldwork. CT, EK and CHM managed data. All authors interpreted data, reviewed successive drafts and approved the final version of the article.

Funding Natsal-3 is a collaboration between University College London, London School of Hygiene and Tropical Medicine, National Centre for Social Research, Public Health England and the University of Manchester. The study was supported by grants from the Medical Research Council (G0701757) and the Wellcome Trust (084840), with contributions from the Economic and Social Research Council and Department of Health.

Competing interests AMJ has been a Governor of the Wellcome Trust since 2011. The other authors declare that they have no conflicts of interest.

Patient consent for publication Not required.

Ethics approval Oxford A MREC.

Provenance and peer review Not commissioned; externally peer reviewed.

Data sharing statement The Natsal datasets are archived with the UK Data Service and are accessible from https://www.ukdataservice.ac.uk/.

Open access This is an open access article distributed in accordance with the Creative Commons Attribution 4.0 Unported (CC BY 4.0) license, which permits others to copy, redistribute, remix, transform and build upon this work for any purpose, provided the original work is properly cited, a link to the licence is given, and indication of whether changes were made. See: http://creativecommons.org/ licenses/by/4.0

\section{REFERENCES}

1 Hughes $\mathrm{G}$, Field $\mathrm{N}$. The epidemiology of sexually transmitted infections in the UK: impact of behavior, services and interventions. Future Microbiol 2015;10:35-51.

2 Patel $H$, Wagner $M$, Singhal $P$, et al. Systematic review of the incidence and prevalence of genital warts. BMC Infect Dis 2013;13:39.

3 Desai S, Wetten S, Woodhall SC, et al. Genital warts and cost of care in England. Sex Transm Infect 2011;87:464-8.

4 Woodhall SC, Jit M, Soldan K, et al. The impact of genital warts: loss of quality of life and cost of treatment in eight sexual health clinics in the UK. Sex Transm Infect 2011;87:458-63.

5 Hawkins MG, Winder DM, Ball SL, et al. Detection of specific HPV subtypes responsible for the pathogenesis of condylomata acuminata. Virol J 2013;10:137.

6 Garland SM, Kjaer SK, Muñoz N, et al. Impact and Effectiveness of the Quadrivalent Human Papillomavirus Vaccine: A Systematic Review of 10 Years of Real-world Experience. Clin Infect Dis 2016;63:519-27.

7 Fairley CK, Hocking JS, Gurrin LC, et al. Rapid decline in presentations of genital warts after the implementation of a national quadrivalent human papillomavirus vaccination programme for young women. Sex Transm Infect 2009;85:499-502.

8 Chow EP, Read TR, Wigan R, et al. Ongoing decline in genital warts among young heterosexuals 7 years after the Australian human papillomavirus (HPV) vaccination programme. Sex Transm Infect 2015;91:214-9.

9 Howell-Jones R, Soldan K, Wetten S, et al. Declining genital Warts in young women in england associated with HPV 16/18 vaccination: an ecological study. I Infect Dis 2013:208:1397-403.

10 Canvin M, Sinka K, Hughes G, et al. Decline in genital warts diagnoses among young women and young men since the introduction of the bivalent HPV (16/18) vaccination programme in England: an ecological analysis. Sex Transm Infect 2017;93:125-8.

11 Szarewski A, Skinner SR, Garland SM, et al. Efficacy of the HPV-16/18 AS04adjuvanted vaccine against low-risk HPV types (PATRICIA randomized trial): an unexpected observation. J Infect Dis 2013;208:1391-6.

12 Erens B, Phelps A, Clifton S, et al. Methodology of the third British National Survey of Sexual Attitudes and Lifestyles (Natsal-3). Sex Transm Infect 2014;90:84-9.
13 Johnson AM, Mercer $\mathrm{CH}$, Erens B, et al. Sexual behaviour in Britain: partnerships, practices, and HIV risk behaviours. The Lancet 2001;358:1835-42.

14 Sonnenberg $\mathrm{P}, \mathrm{Clifton} \mathrm{S}$, Beddows S, et al. Prevalence, risk factors, and uptake of interventions for sexually transmitted infections in Britain: findings from the National Surveys of Sexual Attitudes and Lifestyles (Natsal). The Lancet 2013;382:1795-806.

15 Tanton C, Soldan K, Beddows S, et al. High-Risk Human Papillomavirus (HPV) Infection and Cervical Cancer Prevention in Britain: Evidence of Differential Uptake of Interventions from a Probability Survey. Cancer Epidemiol Biomarkers Prev 2015;24:842-53

16 King EM, Gilson R, Beddows S, et al. Human papillomavirus DNA in men who have sex with men: type-specific prevalence, risk factors and implications for vaccination strategies. Br J Cancer 2015;112:1585-93.

17 King EM. Human papillomavirus epidemiology in men who have sex with men: Implications for a vaccine programme at sexual health clinics in the UK. PhD thesis. 2015. Available: http://discovery.ucl.ac.uk/1473373/12/King_Eleanor_Megan_ THESIS_17DEC15_FINAL.pd.\%20REDACTEDf.pdf

18 Machalek DA, Poynten M, Jin F, et al. Anal human papillomavirus infection and associated neoplastic lesions in men who have sex with men: a systematic review and meta-analysis. Lancet Oncol 2012;13:487-500.

19 Lin A, Ong KJ, Hobbelen P, et al. Impact and Cost-effectiveness of Selective Human Papillomavirus Vaccination of Men Who Have Sex With Men. Clin Infect Dis 2017;64:580-8.

20 Welsh Government. HPV vaccine programme for gay men introduced. 2018. Available: http://gov.wales/newsroom/health-and-social-services/2017/170403hpv/?lang=en [Accessed 25 Apr 2018].

21 HPV MSM. 2018. Available: http://www.immunisationscotland.org.uk/vaccines-anddiseases/hpvmsm.aspx [Accessed 25 Apr 2018].

22 HPV vaccination for men who have sex with men (MSM) programme. GOV.UK.. 2018. Available: https://www.gov.uk/government/collections/hpv-vaccination-for-men-whohave-sex-with-men-msm-programme [Accessed 25 Apr 2018].

23 Mercer $\mathrm{CH}$, Bailey JV, Johnson AM, et al. Women who report having sex with women: British national probability data on prevalence, sexual behaviors, and health outcomes. Am J Public Health 2007:97:1126-33.

24 Tanton C, Mesher D, Beddows S, et al. Human papillomavirus (HPV) in young women in Britain: Population-based evidence of the effectiveness of the bivalent immunisation programme and burden of quadrivalent and 9 -valent vaccine types. Papillomavirus Res 2017;3:36-41.

25 Johnson AM, Mercer $\mathrm{CH}$, Beddows S, et al. Epidemiology of, and behavioural risk factors for, sexually transmitted human papillomavirus infection in men and women in Britain. Sex Transm Infect 2012;88:212-7.

26 Navarro-Illana E, López-Lacort M, Navarro-Illana P, et al. Effectiveness of HPV vaccines against genital warts in women from Valencia, Spain. Vaccine 2017:35:3342-6.

27 Petráš M, Adámková V. Impact of quadrivalent human papillomavirus vaccine in women at increased risk of genital warts burden: Population-based cross-sectional survey of Czech women aged 16 to 40 years. Vaccine 2015;33:6264-7.

28 Woestenberg PJ, King AJ, van der Sande MA, et al. No evidence for cross-protection of the HPV-16/18 vaccine against HPV-6/11 positivity in female STI clinic visitors. J Infect 2017:74:393-400

29 Mesher D, Panwar K, Thomas SL, et al. The Impact of the National HPV Vaccination Program in England Using the Bivalent HPV Vaccine: Surveillance of Type-Specific HPV in Young Females, 2010-2016. J Infect Dis 2018;218:911-21.

30 Kavanagh K, Pollock KG, Cuschieri K, et al. Changes in the prevalence of human papillomavirus following a national bivalent human papillomavirus vaccination programme in Scotland: a 7-year cross-sectional study. Lancet Infect Dis 2017:17:1293-302.

31 Public Health England. Sexually transmitted infections and screening for chlamydia in England, 2017. Health Prot Rep 2018;12.

32 Joint Committee on Vaccination and Immunisation. Statement on HPV vaccination. 2018. Available: https://www.gov.uk/government/publications/jcvi-statementextending-the-hpv-vaccination-programme-conclusions 